\title{
Finding your way through Research Ethics Committees
}

\section{Rachel Smith}

\section{Introduction}

The process of applying to a National Health Service (NHS) Research Ethics Committee (REC) changed in 2004. This was in part to ensure that the UK was fully compliant with the EU Directive for Clinical Trials; however, the changes affect all applications to RECs and not just applications relating to clinical trials. I have tried to outline briefly how the process now works. Knowing the process makes applying a lot less painful.

\section{When do I need to use an NHS REC?}

If you intend to conduct research within the NHS, and you will be involving human subjects (patients, their data, tissue or their carers, NHS staff or the recently dead), you will need to approach an NHS REC for an ethical review. You do not require an ethical review of audits or service evaluations. The difference between research and audit/service evaluation can be subtle. As a guide, if you are intending to collect information that will be directly applicable to others working in a similar field, you are proposing to undertake a piece of research (i.e. your research findings will be generalisable). If you simply want to assess how you, or your immediate colleagues, deliver a specific service, and your findings will not be directly applicable to others outside your immediate clinical area, you are probably planning to conduct an audit or service evaluation. If you are unsure, contact your local REC and they will give you guidance.

\section{What forms do I have to fill in to apply to a REC?}

All NHS RECs in the UK now accept the same form, which is available on the Central Office for Research Ethics Committees (COREC) website. This web-based form does not require you to download or install any files or software onto your own personal computer. You must first register as a user with COREC, a process that only takes a couple of minutes. You will also need to prepare other study-related documentation, including consent forms, patient information sheets, protocols, and so on. A checklist of what accompanying documentation is required is provided with every new form you create. Different checklists are produced by the system, specific to different types of research study (e.g. clinical trial versus collection of tissue).

Perhaps the most critical part of the REC application form to get right is the form filter. The form filter asks you questions about the type of study you are proposing to undertake (e.g. clinical trial, questionnaire study, tissue collection, etc.). Your responses will dictate which questions you will be asked to answer in the body of the form, and the content of the application checklist. If you

J Fam Plann Reprod Health Care 2007; 33(2): 121-122 (Accepted 13 February 2007)

Medical Research Council Regulatory Support Centre, The Queen's Medical Research Institute, Edinburgh, UK Rachel Smith, MSc, PhD, Project Coordinator

Correspondence to: Dr Rachel Smith, MRC Regulatory Support Centre, The Queen's Medical Research Institute, 47 Little France Crescent, Edinburgh EH16 4TJ, UK.

E-mail: r.smith@hrsu.mrc.ac.uk do not understand the questions in the form filter, ask for help; ticking the wrong boxes at this stage will generate an inappropriate form which you will struggle to complete.

The ethics form contains a new site-specific information (SSI) section, which is used by the ethics committee (in assessing the suitability of your specific research site; so-called site-specific assessment or SSA), and by your local NHS Research and Development (R\&D) department (enabling them to conduct a research governance review as part of their R\&D approval process). However, if you are working in Northern Ireland, you will still need to complete a separate form to apply for R\&D management approval.

\section{How do I submit my ethics application?}

You should complete the form apart from the questions asking for administrative details of the committee you are applying to. To determine where you are going to apply for an ethical review, you need ask yourself four simple questions (Box 1). If the answer to any of these questions is yes, telephone COREC's Central Allocation System (CAS). CAS will identify the next meeting available in the UK that is appropriate to review your study. You can ask to go to a different committee if the committee first suggested is not convenient. For all other studies, you should contact your local NHS REC directly. However, if your research involves testing a medical device or building a research tissue bank, you can either submit to your local NHS REC, or you can apply, via CAS, to a REC with a particular interest in such studies. Once you have spoken to your local committee or to CAS you will be able to complete your ethics form. When your form is complete, you should 'lock' it, print a hard copy, sign the hard copy and then send the signed copy together with all supporting documents to the appropriate committee. You must get all your paperwork to the relevant committee before the deadline; if your application arrives too late, you may have to book your application with the local committee or CAS again.

To use the SSI section to apply for R\&D management approval you should submit a copy of this form, together whatever documentation is required by your local $R \& D$ department, to them for consideration.

\section{What about studies with a number of different sites?}

No matter how many sites are going to be involved in a study, you only need one ethical opinion in the UK. However, local ethics committees should assess each site

Box 1: Questions to assist researchers in determining where they should apply for an ethical review

- Do you intend to look at the safety and/or efficacy of a medicinal product? (i.e. Are you proposing to undertake a clinical trial?)

- Do you want to recruit prisoners to your study?

- Do you want to recruit adults with incapacity in Scotland?

- Do you want to involve more than one 'domain'? [A domain being a Strategic Health Authority (England), an NHS Board (Scotland), a regional office of the NHS Wales Department or the whole of Northern Ireland.] 


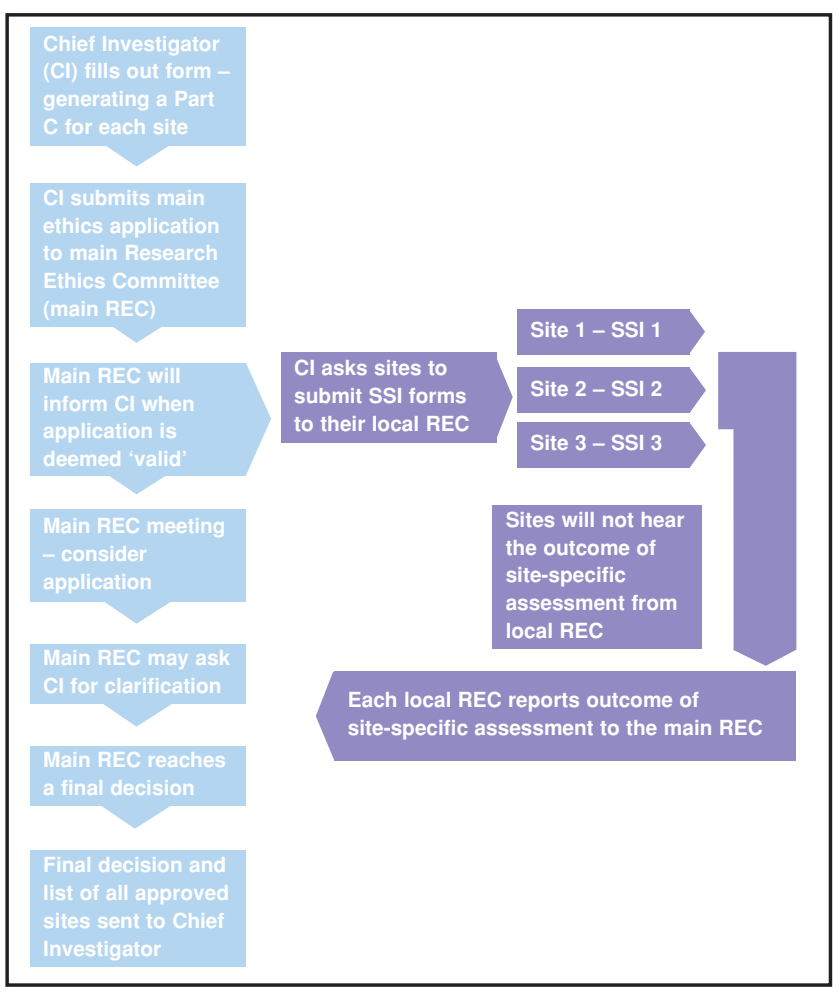

Figure 1 Schematic representation of Research Ethics Committee processes

involved before they take part in the study (Figure 1). Local RECs will conduct all SSAs [for sites in their Strategic Health Authority (SHA)/NHS Board area]. The information on which they base this assessment is contained in the SSI section of the ethics form (one SSI section is required for each site). You can generate as many SSI sections as you need, each being partially populated from the main body of the form. Some simple studies may not require each site to be assessed by the ethics committee; you should check COREC guidance to see if your study falls into this category. However, even if an ethical assessment of each site is not required, each local R\&D department may still wish to review your study.

Box 2: Tips for researchers on applying to an NHS Research Ethics Committee (REC)

- Know your audience - RECs have lay and expert members; use appropriate language

- There are no right or wrong answers - provide honest answers. Don't hide or ignore risks, address them

- Avoid contradicting yourself in your application and enclosures (e.g. patient information sheet)

- Use the standard patient information sheet format (available on the COREC website) and keep all information easily understandable

- Answer every 'active' question on the application form write 'N/A' where appropriate

- Understand questions in the form filter and their implications - ask for help if you are not sure

- Know the process - ask for help if you are not sure

- Go to the meeting if you are able to

- Speak to your collaborators - let them know what is going on

- RECs are there to protect the rights, dignity and well-being of research participants - this is not simply about paperwork and process
Box 3: Sources of information

- COREC website (http://www.corec.org.uk): provides information, guidance as well as the application form itself

- COREC queries line (queries@corec.org.uk): provides timely, specific and reliable information in response to your questions. Do not phone the Central Allocation System with queries; they will not be able to help you

- Research Ethics Committee Administrators (contact details available on the COREC website) and NHS R\&D offices: these can both provide help

\section{What should I expect from the REC?}

Your application will be discussed at a meeting, to which you should be invited. You do not have to attend, but if you are there you can provide clarification or address misunderstandings in person. After the meeting the committee may wish to come back to you for clarification. They can only ask you one set of questions; once you have provided them with adequate answers they must come to a final decision. Occasionally they may wish to ask an expert about the protocol outside of their meeting. Whatever the outcome of the meeting, all RECs must reach a final decision (to approve or not approve) within a maximum of 60 days (not including the time they wait for you to provide clarification). Their final decision will be sent to the Chief Investigator. Investigators at other sites will not hear anything directly from their local ethics committee or from the main REC; they rely on the Chief Investigator to keep them informed of status.

\section{What do RECs look for?}

It is easy to lose sight of what RECs are there to do when you are trying to find your way through the administrative system that serves them. Committees themselves are made up of both expert and lay members, drawn from all walks of life. Their role is to protect the rights, dignity and wellbeing of potential participants in your study. As such they are concerned with the ethical issues that your protocol presents. Committees will spend a considerable time discussing your planned approach to recruiting participants and obtaining their free and informed consent. Central to this will be consideration of the information you intend to provide participants with - ensuring it is appropriate and complete, that it is presented in such a way that participants will be able to fully understand what is being asked of them, and that participants will be approached at a time that allows them to consider fully and freely before making their decision. However, issues around consent and autonomy are not the only ethical considerations made in the review. Committees will also look at the risks you are asking participants to take and consider if these risks are justifiable in the light of what the study aims to achieve. They will ensure that access to the study is as far as is possible equitable, that the study is worthwhile and is likely to produce benefit (even though this benefit may not always be to the participants themselves) and that all appropriate steps are taken to avoid causing harm to participants. Box 2 provides a few tips on applying to an NHS REC. It is by no means comprehensive, but remember that there are a number of sources of help available (Box 3), including your peers who serve on ethics committees.

Statements on funding and competing interests Funding None identified. Competing interests None identified. 